Article

\title{
Understanding the molecular bases of the endophyt- ic/pathogenic behavior of Fusarium oxysporum interacting with Vanilla planifolia
}

\author{
Marco Tulio Solano De la Cruz 1, a, Esteban Elías Escobar - Hernández 2, a , Jorge Arturo Arciniega - González ${ }^{3}$, Ro- \\ cío del Pilar Rueda - Zozaya ${ }^{1}$, Jacel Adame - García ${ }^{4}$, Edel Pérez-López 5, ${ }^{\text {, }}$, Mauricio Luna - Rodríguez 6, ${ }^{*}$ \\ a These authors contributed equally \\ 1 Instituto de Ecología, Universidad Nacional Autónoma de México, Circuito Exterior S/N anexo, Jardín Bo- \\ tánico exterior, Ciudad Universitaria, Ciudad de México, México; msolanodelacruz@iecologia.unam.mx \\ ruedazozayapilar@gmail.com \\ 2 Unidad de Genómica Avanzada, Langebio, Cinvestav, Km 9.6 Libramiento Norte Carretera León 36821, \\ Irapuato, Guanajuato, México; esteban.escobar@cinvestav.mx \\ 3 Centro de Ciencias de la Complejidad, Universidad Nacional Autónoma de México, Ciudad de México, Mé- \\ xico; arturo.arciniegago@gmail.com \\ 4 Tecnológico Nacional de México, Instituto Tecnológico de Úrsulo Galván, Úrsulo Galván, Veracruz, México; \\ jadameg@gmail.com \\ 5 Centre de recherche et d'innovation sur les végétaux (CRIV), Université Laval, Department of Plant Sciences, \\ FSAA, Université Laval; edel.perez-lopez.1@ulaval.ca \\ 6 Laboratorio de Genética e Interacciones Planta Microorganismos, Facultad de Ciencias Agrícolas, Universi- \\ dad Veracruzana. Circuito Gonzalo Aguirre Beltrán s/n, Zona Universitaria, Xalapa, Veracruz, México; \\ mluna@uv.mx

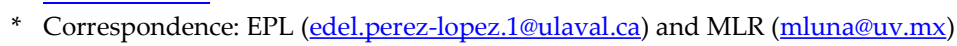

\begin{abstract}
Members of the Fusarium oxysporum species complex (FOSC) has the capacity to specialize into host-specific pathogens known as formae speciales through horizontal gene transfer between pathogenic and endophytic individuals. To this day, the origin of these formae speciales and the genetic determinants dictating the switch from endophytic to pathogenic Fusarium oxysporum (Fox) are still unknown. F. oxysporum f. sp. vanillae (Fov), member of FOSC, is the causal agent of root and stem rot disease, representing the main phytosanitary problem in vanilla plantations worldwide. Here we analyzed the RNA-seq libraries resulting from the interaction vanilla-Fov at early and late stages of the infection, and what we initially identified as control in a previous study, detecting the presence of Fox endophytes. We identified virulence, hypervirulence, sporulation, conidiation, necrosis, and production of fusaric acid as key processes taking place during Fov-vanilla interaction. Through comparison with endophytic Fox, we found that Fov can infect vanilla thanks to the presence of pathogenicity islands and genomic regions associated with supernumerary chromosomes. These play a central role as carriers of genes involved with pathogenic activity and could have being obtained by Fov through horizontal gene transfer. We also found that, unlike other pathogenic members of FOSC, Fov do not use Secreted in Xylem proteins (SIX) to infect vanilla.
\end{abstract}

Keywords: formae speciales; horizontal gene transfer; behavior; endophytic; pathogenic; Fusarium; RNAseq; comparative genomics; vanilla; Mexico

\section{Introduction}

Vanilla is the second more expensive spice and one of the most used worldwide [1]. This spice is native to Mexico and comes from the orchid Vanilla planifolia Jacks., now widely cultivated in Asia and Oceania [1]. Mexico currently occupies the third place among the main producers in the world, although vanilla production has seriously suffered from the appearance of synthetic products that are easier to produce [2,3]. Several factors can limit vanilla production, but the main phytosanitary problem, putting at risk 
the vanilla industry worldwide, is root and stem rot disease caused by Fusarium oxysporum f. sp. vanillae (Fov) [4-6].

Fov has been reported in different vanilla producing regions around the world, however, its origin and evolution are poorly understood [4]. The fungal genus Fusarium contains more 300 phylogenetically soilborne distinct species/species complexes [7], including pathogenic and non-pathogenic strains. Within the Fusarium oxysporum species complex (FOSC) exist a broad genomic plasticity. Many of the pathogenic strains, due to their narrow host specificity, have been identified as formae speciales, each grouping strains with the same host range, generally the same plant species [8].

For a long time was believed that members from the same formae speciales shared genomic structure and the same evolutionary origin [10-12], but recent studies have pointed that some formae speciales like Fov have multiple independent origins [13], evidence of genomic plasticity. This genetic diversity into the formae speciales is also evidenced by the fact that some members can live in the host as endophyte without inducing symptoms, while others are pathogenic, but what makes them pathogenic? Is mainly in the pathogenic strains that the presence of certain supernumerary chromosomes, like chromosome 14, has been reported, phenomenon associated with the shift from non-pathogenic to pathogenic of FOSC members [9]. However, the presence of supernumerary chromosomes alone does not explain what makes certain members of Fov pathogenic and what makes them endophyte.

To answer this question our group analyzed the RNA-seq data obtained from the interaction of Fov with V. planifolia at 2- and 10-days post inoculation (dpi). This data was previously obtained by our group [14]. However, here for the first time, we were able to identify that in the treatment used as control by our group, there were endophytic Fox, allowing to establish comparisons between pathogenic Fov and endophytic Fox during vanilla colonization. Through the analysis of the RNA seq, we have been able to identify the main molecular mechanisms used by Fov to infect vanilla and to gain pathogenicity.

\section{Materials and Methods}

2.1 Experimental framework

The experimental framework, that includes plant material collection, infectivity assays, total RNA extraction, generation of cDNA libraries, and sequencing details, is explained in detail in Solano-De la Cruz et al. [14]. Raw data was submitted to the GEO platform of NCBI-GenBank under the accession number: GSE134155.

2.2 RNA-seq quality control, splice-aware mapping and alignment filtering

The quality control of the reads obtained from the high-throughput sequences was carried out using FASTQC version 0.11.2 [15], and MultiQC version 1.0 [16] software. Reads above $32 \mathrm{nt}$, without the presence of adapters were considered for further analysis. First, to filter and discard reads corresponding to the plant host, genome index and alignment of reads was done utilizing the STAR program version 2.7.2 [17] using the reference genome of F. oxysporum f. sp. lycopersici strain Fol 4287 available on Ensembl Fungi, visualizing the alignment output with MultiQC version 1.0 software [16]. To further filter the reads based on alignment quality, Samtools version 1.9 [18] and MultiQC for post-filtering visualization, were used.

\subsection{Phylogenetic analysis}

To investigate the identity of the Fusarium spp. sequences found in the control, we searched for the reads mapping EF-1a. Both, control and treatment EF-1a genes were aligned and compared with reference sequences from GenBank using BLAST (www.ncbi.nlm.nih.gov). A global sequence alignment was reconstructed using the CLUSTAL_W option of the MEGALIGN and a phylogenetic tree was constructed using the neighbor-joining method available in Mega version X [19]. 
2.4 Genes identification and visualization of Fov genomic data

To be able to identify Fov transcripts participating in the infection, the intersection of the genomic coordinates between F. oxysporum f. sp. lycopersici strain Fol 4287 genome and the filtered reads was done using Bedtools version 2.25.0 [21]. This allowed the extraction of genes ID using cut command and AWK. The bed files with the genomic coordinates were used to map and visualize the Fov genomic data in Fol 4287 karyogram template using the visualization tool from Ensembl Fungi of Ensembl Genomes consortium [21] (https://fungi.ensembl.org/Fusarium_oxysporum/Location/Genome).

\subsection{Enzyme annotation and metabolic reconstruction}

To elucidate the metabolic pathways involving the genes identified, the annotation of enzyme coding sequences and metabolic reconstruction of these biochemical processes were conducted. First, we extracted the protein sequences using the Biomart-Ensembl Fungi database [21] to annotate them with the BlastKoala tool from KEGG platform (https://www.kegg.jp/kegg/tool/annotate_sequence.html). Later, using the annotations, the metabolic reconstruction was performed the species-specific KEGG pathway maps of F. oxysporum as reference and the Search Pathway tool from KEGG platform (https://www.genome.jp/kegg/tool/map_pathway1.html) [22].

\subsection{Gene Ontology Enrichment Analysis}

GO enrichment analysis was executed using the topGO R package [23]. We applied the following criteria: $p$-value $<0.05$ threshold, algorithm "classic" and "fisher" statistic. As query we used the GeneIDs lists obtained through the comparison with F. oxysporum f. sp. lycopersici strain Fol 4287. Finally, the data obtained was visualized via RStudio version 4.0.1 and ggplot2 package version 3.3.2 [24].

\subsection{KEGG Gene Enrichment Analysis}

To identify the biological processes overexpressed during the infection, we performed the KEGG enrichment analysis using RStudio and the clusterProfiler package [25]. To use the enrichKEGG function in this package, the input were the KO identifiers obtained from BlastKoala annotation with the results filtered applying adjusted $p$-value cut-offs $<0.05$. For the visualization we used RStudio version 4.0.1 and ggplot2 $\mathrm{R}$ package version 3.3.2 [24].

\subsection{De novo annotation of Fov genes}

To identify the potential functions of Fov transcripts we used the software InterproScan 5 version 5.41 [26]. Which allows protein-coding sequences function prediction, using as reference databases Pfam (http://pfam.xfam.org/) and SUPERFAMILY (https://supfam.org/).

\section{Results}

3.1 Identification of F. oxysporum endophyte in vanilla

The quality analysis of the libraries corresponding to the V. planifolia transcriptome in response to the F. oxysporum f. sp. vanillae infection, was successfully performed and confirmed our previous reports [14]. A surprising finding for us was the detection of $F$. oxysporum reads in the mock-inoculated control treatments from our previous study [14]. The alignment of the libraries against the Fol 4287 genome was performed, obtaining from the control libraries, at 2 dpi 7158305, 7249959, and 5741789 reads for each replicate, respectively, while at $10 \mathrm{dpi} 6458564,6730883$, and 6528340 reads. In $2 \mathrm{dpi}$, a total of 10917618,10817677 , and 10757914 aligned to Fol 4287 reads were collected, and at $10 \mathrm{dpi}$ 9397911, 10190151, and 10190151, reads were retrieved. Next, we filtered the alignments results by alignment quality and sorted them by genomic coordinates. 
To study the phylogenetic relationship between Fov and what we believe is a Fox endophyte colonizing vanilla, a neighbor-joining analysis was performed. The results clearly indicated that the sequence detected in the control is indeed from members of $F$. oxysporum, and it did not group with Fov (Fig. 1). We were not able to establish the specific taxonomy of the endophytes, but this analysis clarified that we are not in the presence of Fov in the control samples, but in the presence of endophytes Fox.

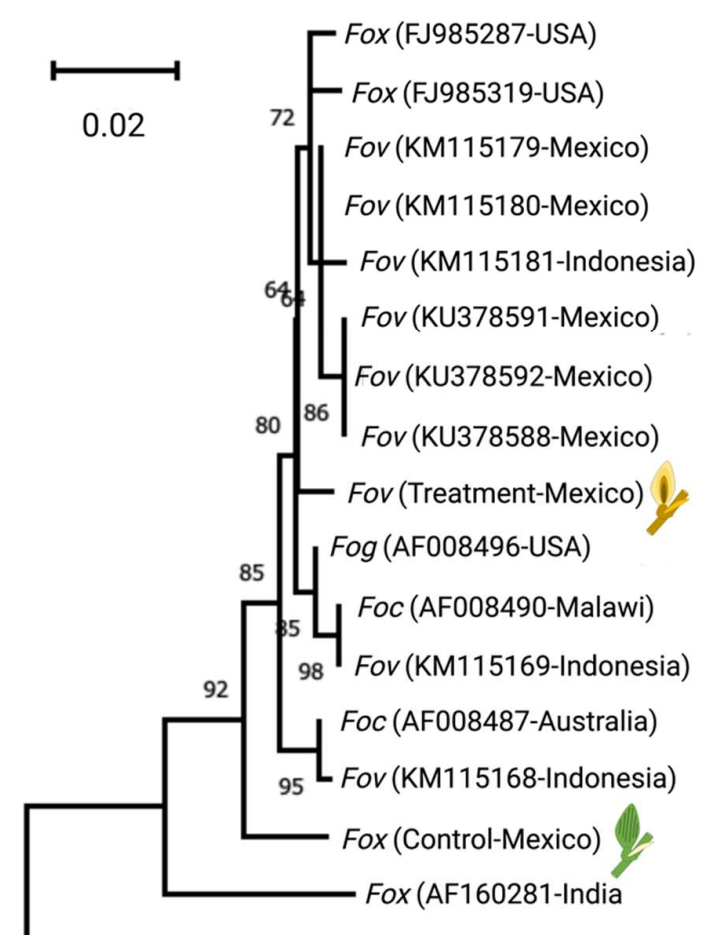

F. solani (DQ247675-Germany)

Figure 1. Phylogenetic tree reconstructed through the neighborhood-joining method of the EF-1a gene sequences from the treatment (Fov) and control (endophytic Fox) and sequences retrieved from Genbank. Accession numbers and country of the strains are indicated in parentheses. Fusarium solani was used as an outgroup in the tree. The phylogenetic tree was bootstrapped 1000 times. Bar, 2 substitution in 100 positions.

3.2 Fov genomic data visualization revealed the presence of supernumerary chromosomes

The genes coded by the reads retrieved at 2-and 10- dpi from controls and treatments were identified using Fol 4287 the genomic coordinates (Supplementary Table 1). These genes were then mapped to Fol 4287 chromosomes finding that most of the transcripts corresponding to endophytic Fox were mainly located in the core chromosomes of Fol 4287, reference genome for Fox formae speciales (Fig. 2). At 2 dpi, we observed the presence of transcripts corresponding mainly to chromosomes 1, 2, 4, 5, 7, 8, 9 and 10 with the presence of some transcripts in chromosomes 3, 6, 11, 12, 13 and 14, while no transcript from chromosome 15 was detected (Fig. 2). At $10 \mathrm{dpi}$, we detected transcripts from chromosomes 1, 2, 4, 5, 7, 8, 9 and 10, with less, but still present, from chromosomes 3, 11, 12 and 13. Interestingly, transcripts related to chromosomes 6,14 , and 15 were absent (Fig. 2).

The number of transcripts resulting from the interaction Fov-vanilla (treatment) was higher than the control, and after mapping we identified that they are not only from genes located in the core chromosomes, but also in the supernumerary chromosomes (Fig. 2). These was clear at 2 dpi after comparing control with treatment, showing a higher number of transcripts in core chromosomes 1, 2, 4, 5, 7, 8 and 9 (Fig. 2), and in the 
supernumerary chromosomes associated with pathogenicity in Fox 10, 11, 12, 13 and 14 (Fig. 2). This was consistent at $10 \mathrm{dpi}$, with a high number of transcripts located in chromosomes 10 to 15 during the interaction Fov-vanilla (Fig. 2). These results are strong evidence of the presence of supernumerary chromosomes in Fov, something that until this day had remained unknown.

A

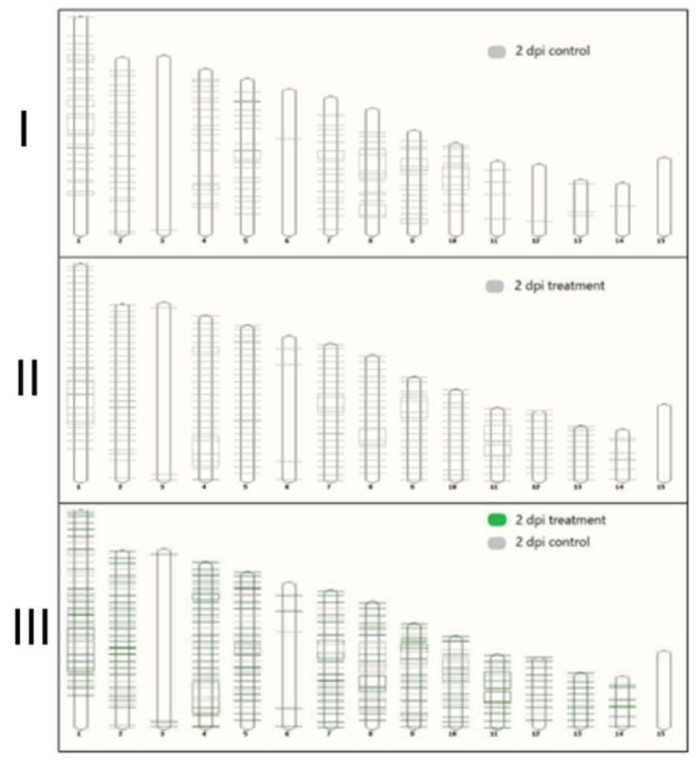

B

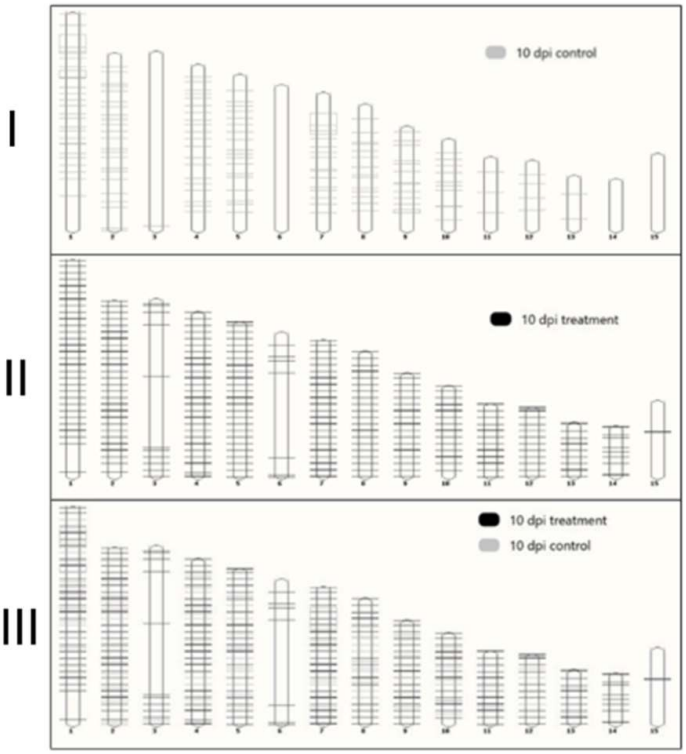

Figure 2. Mapping of transcripts obtained from the interaction of endophytic Fox-vanilla (control) and Fov-vanilla (treatment) using as reference the 15 chromosomes of Fol 4287 karyogram. A. Reads obtained at 2 dpi. B. Reads obtained at $10 \mathrm{dpi}$. Panel I represent the reads obtained for the control, panel II the treatment, and panel III the superposition of the reads obtained for the control and the treatment.

In addition to the presence of supernumerary chromosomes in Fov, we also identified in the treatment reads that correspond to Fox pathogenicity island component (Fig. 3). This sequence was detected at $10 \mathrm{dpi}$ in the treatment only, not present in the meta transcriptome data obtained from the interaction vanilla-endophytic Fox.



Figure 3. Visualization of the treatment Fov (red tracks) at $10 \mathrm{dpi}$, its control (green tracks) and the gene structure (blue tracks) in the genomic coordinates of Fol 4287 corresponding to a Pathogenicity Island Component. 
3.3 Metabolic pathways Fov associated with $V$. planifolia infection

After annotating the genes present in the reads retrieved from the interaction endophytic Fox-vanilla and Fov-vanilla, we performed a functional prediction annotation and metabolic mapping using KEGG database [21] (Supplementary dataset 1). We also identified the number of genes having a role in each of the pathways at 2 and $10 \mathrm{dpi}$ (Supplementary Table 2, and Supplementary dataset 2). An interesting finding was that the number of genes corresponding to each metabolic pathway identified was considerably higher during late infection compared to early infection.

At 10 dpi we determined the presence of genes corresponding to the following metabolic pathways, only found during late infection: penicillin and cephalosporin biosynthesis, homologous recombination, biotin metabolism, D-Arginine and D-ornithine metabolism, atrazine degradation, synthesis and degradation of ketone bodies, sulfur relay system, C5-branched dibasic acid metabolism, polyketide sugar unit biosynthesis; carotenoid biosynthesis, seleno compound metabolism, vitamin B6 metabolism, carbapenem biosynthesis, sesquiterpenoid and triterpenoid biosynthesis (Supplementary dataset 1).

3.4 Gene Ontology and KEGG Gene Enrichment offer crucial insights about Fov pathogenesis in $V$. planifolia

Through GO analysis, we obtained 43 and 125 statistically significant biological processes related with pathogenicity at $2 \mathrm{dpi}$ and $10 \mathrm{dpi}$, respectively. At $2 \mathrm{dpi}$ we identified transcripts involved on sporulation such as cell wall biogenesis and external encapsulating structure organization. Also, mechanisms possibly linked to virulence and infection, like protein, peptide, and organic substance transport (Fig. 4A). At 10 dpi we identified mostly regulatory mechanisms of nucleic acids, metabolic processes of macromolecules and organic substances, and mechanisms related to sporulation and cell cycle (Fig. 4B). These results suggest that pathogenic mechanisms key for the pathogen infection remain active along the infection process from early to late stages. All these biological processes were only found in Fov.
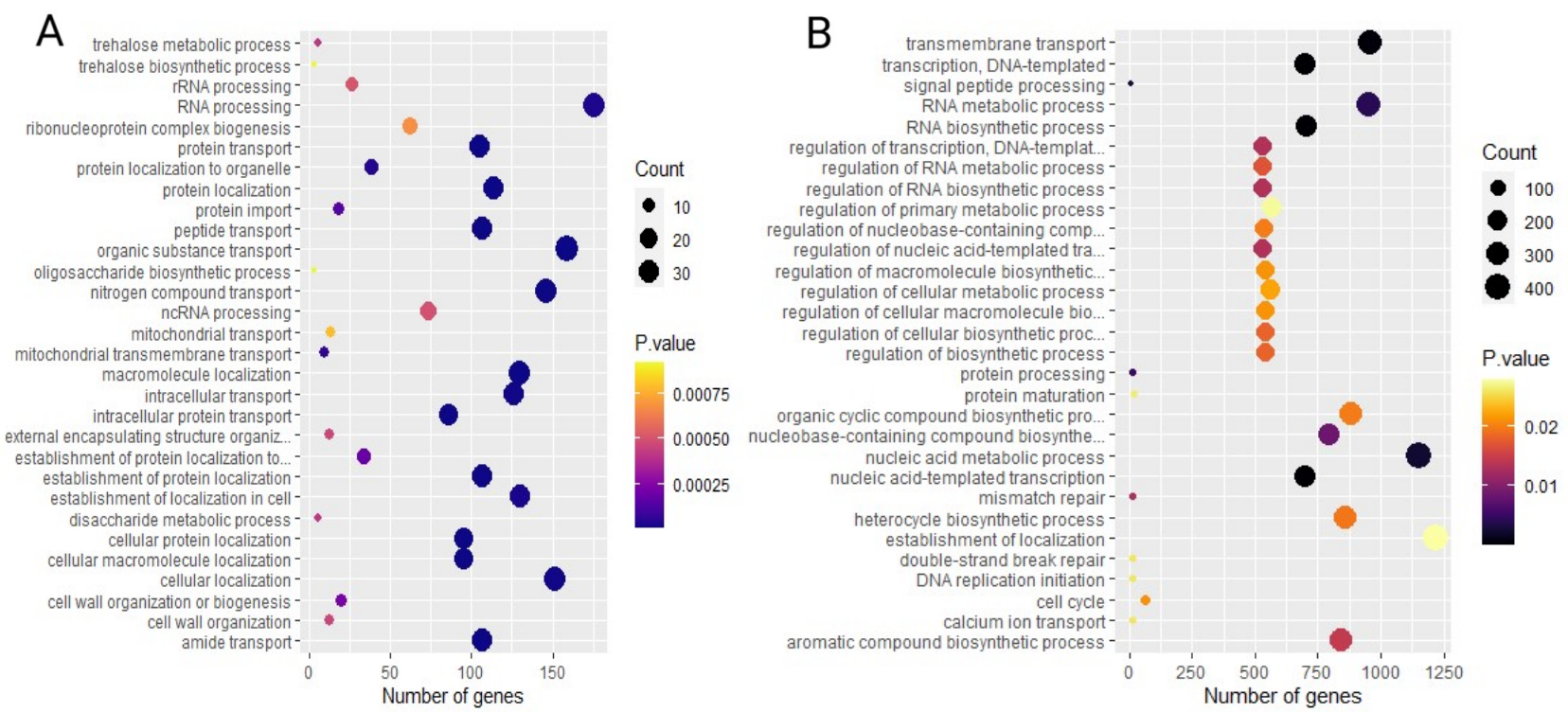

Figure 4. Gene Ontology of top 30 biological processes of Fov activated during the interaction with V. planifolia. A. At 2dpi. B. at $10 \mathrm{dpi}$. 
Regarding the metabolism, we found 21 and 46 statistically significant pathways at 2 dpi and $10 \mathrm{dpi}$, respectively. At 2 dpi we observed pathways involved on reproduction and biosynthesis of metabolites and other biomolecules (Fig. 5A). Similarly, we found pathways linked with the same processes at $10 \mathrm{dpi}$ (Fig. 5B). Additionally, there were other types of biomolecules synthesized at the late stage of infection like cofactors and amino acids that might be used by Fov during the necrotrophic infection. These results further confirm the GO analysis where biological processes, and now metabolic pathways are associated with pathogenesis and stay operating through the infection.
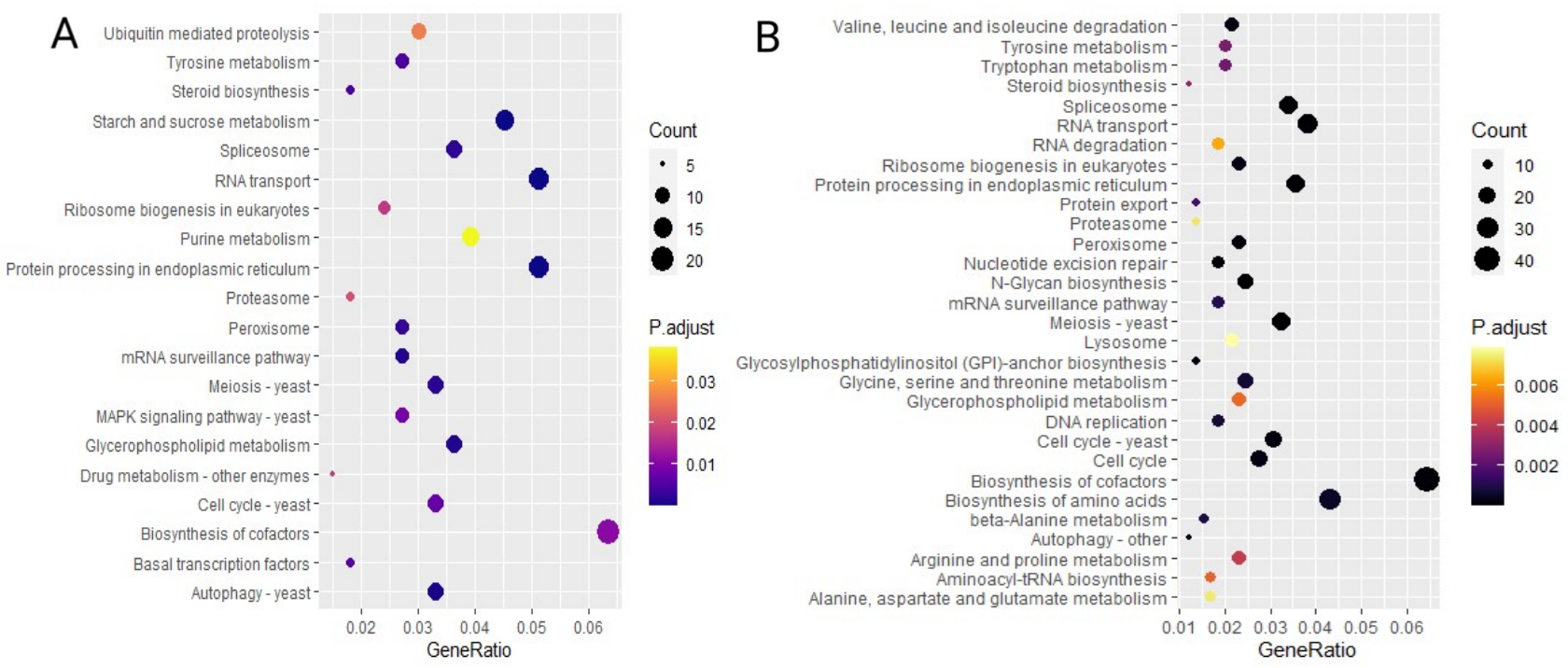

Figure 5. KEGG Gene Enrichment Analysis of top 20 and top 30 metabolic pathways of Fov activated during the interaction with $V$. planifolia. A. At 2 dpi. B. at $10 \mathrm{dpi}$.

3.5 De novo annotation of Fov transcripts shed light on the role of pathogenesis related genes in the plant-host interaction

To characterize the molecular components that may play a main role during the pathogenesis in vanilla, we did a de novo annotation as a functional prediction of Fov genes. For that, we organized the genes on shared or not shared between early and late Fov infection (Supplementary Table 2). Among the genes annotated, we found virulence and hypervirulence factors during both stages of infection. Interestingly these virulence factors increased in number and types during the late stage of infection (Supplementary Table 3), in comparison with the early stage of infection (Supplementary Table 4). This could mean that different virulence factors participate during different time frames along the interaction Fov-vanilla.

At 2 dpi we obtained genes associated with sporulation, necrotic/pathogenic activity and fusaric acid production (Supplementary Table 5-7). These results further confirm the molecular mechanisms part of the pathogenic toolbox used by Fov during early stages of the infection. At $10 \mathrm{dpi}$ we found that the genes involved in the pathogenic processes at 2 dpi were also over expressed but the pathogen also use at this moment effector proteins to facilitate the infection (Supplementary Table 8). Here we found that conidiation, expression of putative effector proteins, and production of fusarinine were active (Supplementary Table 9 and 10). This analysis allowed the identification of processes taking place exclusively late during the interaction Fov-vanilla, and not present during the interaction endophytic Fox-vanilla.

\section{Discussion}


F. oxysporum is the most cosmopolitan species of Fusarium worldwide, and the most commonly reported endophyte in wild and agricultural crops [27, 28]. Through the analysis of the RNAseq resulting from F. oxysporum f. sp. vanilla - Vanilla interaction and the healthy control [14], surprisingly for us, we found Fusarium spp. reads in the control. After analyzing the reads we were able to confirm that belonged to a F. oxysporum not grouping phylogenetically with other forma specialis or to Fov strains previously found in Mexico [29]. Here we considered that Fox detected in the control as an endophyte, based on the recently expanded endophyte concept [30]. Around 20 species of the genus Fusarium have been isolated from the root of $V$. planifolia in Indonesia, but F. oxysporum was the dominant species among those isolates, representing the $55.72 \%$ of the total (542 isolates) [12]. However, all Fox species analyzed in Indonesia were pathogenic, further identified as Fov [12]. On the other hand, in Reunion Island, among 12 different Fusarium species associated with vanilla, two were Fox, and only one pathogenic [31]. Although it is true that most of these isolates are potentially pathogenic there are Fox isolates that do not have the capacity to be pathogenic [32]. One of the main differences among pathogenic Fox and endophytes are the colonization strategies. Is well documented that Fox endophytes are mostly root and bark surface colonizers. Extensive colonization of the root cortex and vasculature is generally restricted to pathogens, a property that correlates with increased secretion of cell wall-degrading enzymes by these strains [33]. Pathogenic strains can also enter and, to some extent, colonize the vascular vessels of a resistant host.

The main difference between Fox pathogenic and non-pathogenic strains is the presence of supernumerary chromosomes. For example, the pathogenicity of Fol in tomato is directed by the presence of chromosome 14 [9], and there is evidence that horizontal transfer between of this chromosome from a pathogenic isolate to an endophyte, also transferring the pathogenic capability to the endophyte Fox [9, 34]. These chromosomes involved on the pathogenicity are characterized by a high content of transposable elements and a low genetic density [9]. In Fol, the pathogenicity chromosome carries the genes that encode putative effector proteins secreted by the pathogen in tomato xylem sap after infection [35]. Some of these xylem secreted proteins, or SIX, have been fully described in the pathosystem Fol-tomato as effectors that contribute to the infection, such as Avr3, a small cysteine rich protein recognized by the plant and key for I-3 mediated resistance in tomato [36]; Avr2, also known as SIX3, a virulence factor that triggers immunity in I-2 carrying tomato plants [37]; Avr1 or SIX4, a small secreted protein found to suppress the activity of two disease resistance genes of tomato [38]; and SIX6, an effector that contributes to virulence and suppresses I-2 mediated cell death in tomato plants [39]. It is important to mention that most of these effectors were identified to be Fol strain specific. To this day, none of these SIX proteins or similar effectors have been identified in the genus of vanilla-related special forms. Therefore, some pathogenicity mechanisms not dependent on these virulence factors have been suggested for Fov.

The synthesis of carbohydrates, as well as the production of sugars, alcohol and organic acids are some of the most active metabolic pathways of Fox metabolism during the germination process of conidia [40]. Indeed, our results showed that these pathways are the most differentially expressed between endophytic Fox found in vanilla and Fov infecting vanilla. The expression of genes related to the synthesis of carbohydrates, production of alcohol and organic acids in the treatments with Fov was detected early and late during the infection. We also showed the presence of transcripts related to carbohydrate metabolism, oxidative phosphorylation, glycolysis, and the pentose phosphate pathway, metabolic pathways related to the germination of Fox conidia [41]. At 2 dpi we found the expression of genes coding hexokinase, trehalose 6-phosphate synthase, beta-fructofuranosidase, neutral trehalase, murein transglycosylase, alpha-trehalose-phosphate synthase, trehalose 6-phosphate synthase, endoglucanase type B, beta-glucosidase, glucoamylase, alpha-amylase, glycosyl hydrolase family, all corresponding to the metabolism of starch and sucrose (Supplementary dataset 1 and 2). The above is evidence of the use of an energy source during Fov infection in vanilla root, which previous works have associated with the growth of hyphae in Fox during the 
germination of conidia, a biological process that is associated with the early stages of infection.

At 10 dpi we found the following genes linked to the metabolism of starch and sucrose highly expressed during Fov-vanilla interaction: beta-glucosidase, murein transglycosylase, glucan 1,6-alpha-glucosidase, beta-glucosidase G, trehalase, beta-glucosidase, alpha-glucosidase, endoglucanase, beta-glucosidase $\mathrm{K}$, glycogen phosphorylase, endoglucanase, oligo-1,6-glucosidase, alpha alpha-trehalse, glucan 1,3-beta-glucosidase. In addition to the genes involved with glycolysis and gluconeogenesis: alcohol dehydrogenase $(\mathrm{NADP}+)$, aldehyde dehydrogenase $(\mathrm{NAD}+)$, pyruvate decarboxylase, and aldose 1-epimerase. This shows the involvement of carbohydrate metabolism in the late phase of Fov infection with vanilla.

The role of amino acid metabolism in plant-pathogen interaction and in the establishment of pathogenicity has been previously documented [42-46]. At 2 dpi and 10 dpi, different genes related to amino acid biosynthesis were found expressed during Fov-vanilla. It has been reported that the inhibition of amino acid biosynthesis has an antifungal activity reducing Fox growth [47]. At 10 dpi we observed transcripts related to arginine and proline metabolism such as glutamate 5-kinase; 1-pyrroline-5-carboxylate dehydrogenase; ornithine aminotransferase; 1-pyrroline-5-carboxylate dehydrogenase; N1-acetylpolyamine oxidase; N1-acetylpolyamine oxidase; amidase; ornithine decarboxylase; agmatine deiminase; and polyamine oxidase. In general, arginine and proline metabolism has been associated with fungal pathogenicity, existing evidence that during F. graminearum infection, these processes are active [48].

Protein metabolism is also key in the establishment of pathogenicity. We found at 2 dpi the presence of genes associated with the processing of proteins in the endoplasmic reticulum, such as nuclear protein localization protein 4, protein disulfide-isomerase erp38, translocation protein SEC63, protein transporter SEC23, and DnaJ-like subfamily B. Also, in the treatment of $2 \mathrm{dpi}$ we detected the presence of genes linked to proteolysis mediated by ubiquitination in Fov transcriptome. These genes correspond to F-box and WD-40 domain-containing proteins MET30 and CDC4, SUMO-conjugating enzyme ubc9, ubiquitin-like 1-activating enzyme E1 B, and ubiquitin-conjugating enzyme E2 R. Recently, numerous studies revealed that F-box proteins are required for fungal pathogenicity [49]. The function of F-box proteins and their potential as part of the ubiquitin-proteasome system has been reported in several fungal pathogens, including those that cause human infections such as Cryptococcus neoformans and Candida albicans or plant diseases such as Fusarium spp. and Magnaporthe oryzae [49]. Fusarium spp. F-box proteins repertoire is larger than in yeast, with approximately 60-94 compared to around 20 in Saccharomyces cerevisiae, C. albicans, or C. neoformans, suggesting that F-box proteins contribute to the regulation of more complex developmental and metabolic processes occurring in filamentous fungi [49].

Autophagy has been described as one of the main routes of cell traffic and recycling, also playing a relevant role in pathogenesis [50]. It has been reported, through genome-wide analysis, that the wheat pathogen F. graminearum has 28 autophagy-related genes, some necessary asexual and sexual differentiation [40]. At 2 dpi we found the presence of genes involved with the autophagy overexpressed. Recently was found that autophagy can affect the morphology, development, improvement, and pathogenicity of Fox [51]. In the present work we found at 2 dpi genes related to the glycerophospholipid metabolism pathway overexpressed like acetyltransferase, phosphatidylserine decarboxylase, phosphatidylserine decarboxylase, and ethanolamine-phosphate cytidylyltransferase. During late infection, we found that more glycerophospholipid metabolism pathways were overexpressed, pointing to their role on pathogenicity, in addition to development. Lipid metabolism has such an important role for fungal development and pathogenicity that has been suggested as a target for antifungal compounds [52].

The infection has been detailed described for F. oxysporum f. sp. lycopersici. Its pathogenic process is divided into four main events. It starts with the hyphae adhesion to the plant surface, followed by the penetration of the plant structure which depends on biotic 
and environmental factors, considering that Fol is an opportunistic pathogen that needs an injury to enter [53]. Once the fungus is established in the plant, the colonization process will begin. The mycelium and spores advance towards xylem vessels causing conidiation to start, for later being transported with the xylem sap through the plant. Finally, the pathogen causes the occlusion of xylem vessels and hydric stress by the accumulation of mycelium and toxins leading to a variety of symptoms including vascular wilt, abscission, chlorosis, and necrosis [54-57].

In vanilla, there exists few reports of F. oxysporum f. sp. radicis-vanillae affecting this orchid. The symptoms start with the browning and death of underground and aerial roots, continuing with wrinkles in leaves and stem and ending with the death of the plant. The infection starts with the hyphae adhesion, a process that takes two days to happen. After eight days the colonization of the roots is complete and the plant start to exhibit the symptoms characteristics of the disease leading to the death $[58,59]$.

In this study we report at $2 \mathrm{dpi}$ the expression of genes related to sporulation, fusaric acid, necrosis, virulence and hypervirulence factors, explaining the beginning of conidia germination and establishment of the infection. These pathogenesis-related genes act as key players during this early plant-host interaction. In concordance, with progression of plant colonization, the plants experiment softening of the root tissue as consequence of fusarium wilt. These symptoms could be justified with the data shown at $10 \mathrm{dpi}$, where we found the overexpression of genes related to conidiation, pathogenic effectors and fusarinine in addition to those reported at $2 \mathrm{dpi}$. These are mainly linked to pathogenesis and cell death, exhibiting a predominant necrotic behavior as reported in this formae specialis [59]. Based on the results, the possible pathogenic strategy of Fov was summarized in figure 6.

Growing evidence supports the hypothesis that pathogenicity of FOSC relies on transferable extra chromosomes and genes coding for Secreted in Xylem proteins also known as SIX [34-36, 59]. Intriguingly, although we found the presence of pathogenicity chromosomes and pathogenicity islands, we did not find SIX genes overexpressed during Fov infection, suggesting a SIX-independent infection strategy.

Figure 6. Hypothetical model of the F. oxysporum f. sp. vanillae pathogenesis

\section{Early stage of infection strategy of $F$. oxysporum f. sp. vanillae}

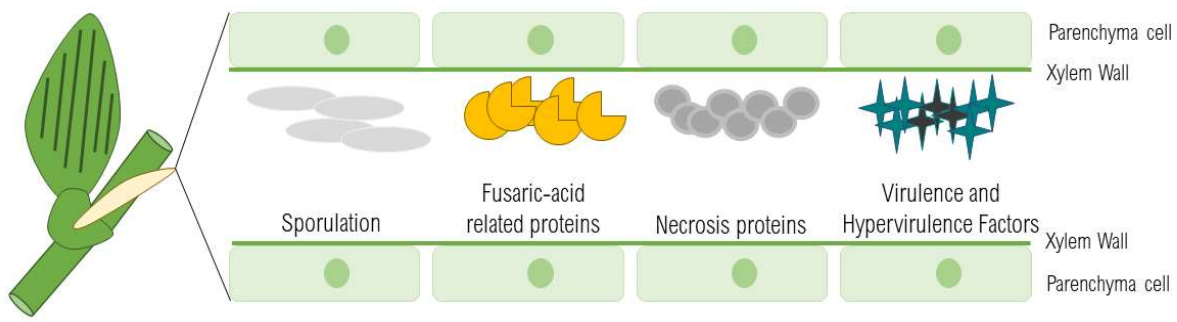

5.



\section{Conclusions}

The bioinformatic analysis of Fov transcripts during the infection in V. planifolia revealed that this formae specialis use different pathogenesis associated genes along this process. Among them we must remark sporulation, conidiation, fusaric acid, necrosis, pathogenic and virulence effectors as main molecular players. Events that are associated with primary metabolism, carbohydrates, amino acid metabolism, autophagy, and sec- 
ondary metabolism. This study opens the door to better understanding the interaction Fov-vanilla and to have clear targets for wet lab characterization. Another important finding presented here was the identification of a pathogenicity component in Fov genome but not in endophytic Fox, which could be used as a molecular marker to detect pathogenic Fov affecting vanilla or to monitor the soil and to avoid outbreaks. All these should be explored in further studies. The results shown here highlight the key players that confer the capacity to infect vanilla to the deadliest pathogen affecting this crop.

Supplementary Materials: The following are available online at www.mdpi.com/xxx/s1, Dataset 1: Metabolic routes, active by timepoint, Dataset 2: Genes overexpressed by timepoint, Table 1: Fusarium oxysporum f. sp. vanillae reads filtered using Fol 4287 genome as reference, Table 2: Number of reads of each timepoint and reads shared between 2 and $10 \mathrm{dpi}$, Table 3: Hypervirulence and virulence factors of Fov at $2 \mathrm{dpi}$, Table 4: Hypervirulence and virulence factors of Fov at $10 \mathrm{dpi}$, Table 5: Sporulation of Fov at 2 dpi, Table 6: Necrosis/pathogenesis of Fov at 2 dpi, Table 7: Fusaric acid production of Fov at 2 dpi, Table 8: Conidiation and sporulation of Fov at 10 dpi, Table 9: Necrosis/pathogenesis of Fov at $10 \mathrm{dpi}$, and Table 10: Fusaric acid production of Fov at $10 \mathrm{dpi}$.

Author Contributions: Conceptualization, MTSC, EEEH and MLR; methodology, MTSC and EEEH; formal analysis, MTSC, EEEH, RPRZ, JAAG and EPL; resources, JAG, MLR and EPL.; writing-original draft preparation, MTSC, EEEH, MLR, RPRZ, and ELP; writing-review and editing, MTSC, EEEH, MLR, and EPL; supervision, MTSC and MLR; funding acquisition, JAG, MLR and EPL. All authors have read and agreed to the published version of the manuscript.

Funding: This research was funded by Tecnológico Nacional de México (project number 5911.16-P Transcriptome of Vanilla planifolia Jacks., exposed to infection caused by Fusarium oxysporum f. sp. vanillae) and by NSERC/CRSNG through the Discovery Grant awarded to Edel Pérez López.

Data Availability Statement: The datasets generated and/or analyzed during the current study are available in the GEO repository with accession number GSE134155 (https://www.ncbi.nlm.nih.gov/geo/query/acc.cgi?acc=GSE134155).

Acknowledgments: MTSC gratefully acknowledges to Dr. Rosalinda Tapia López for reviewing original draft preparation. To the University Massive Sequencing Unit of the Institute of Biotechnology, UNAM. To M.C Jerome Jean Verleyen for his help in the use of the Teopanzolco bioinformatics cluster. To the National Council of Science and Technology (CONACYT), for the scholarship 720145 granted to the first author EEEH to carry out his postgraduate studies. To the National Laboratory of Genomics for Biodiversity (LANGEBIO), CINVESTAV for the MAZORKA bioinformatic cluster services. And to the Tecnológico Nacional de México for financial support of this work.

Conflicts of Interest: The authors declare no conflict of interest. The funders had no role in the design of the study; in the collection, analyses, or interpretation of data; in the writing of the manuscript, or in the decision to publish the results.

\section{References}

1. Hernández-Hernández, J. Vanilla Diseases. In: Handbook of Vanilla science and technology. London: Wiley-Blackwell Publishing, United Kingdom, 2011; pp. 27-39. Doi: https://doi.org/10.1002/9781119377320

2. FAOSTAT. Vanilla production in 2018; Production/Crops/Production Quantity from Elements. UN Food and Agriculture Organization, Statistics Division. 2018. Retrieved 6 May 2020.

3. El Financiero. Producción de vainilla en México, a punto de 'esfumarse' ante productos sintéticos. 2021. Retrieved 22 May 2021.

4. Pinaria, A.; Laurence, M.; Burgess, L.; Liew. E. Phylogeny and origin of Fusarium oxysporum f. sp. vanillae in Indonesia. Plant Pathol. 2015, 64, 1358-1365. Doi: https://doi.org/10.1111/ppa.12365

5. Tombe, M.; Kobayashi, K.; Ogoshi, A. Vegetative compatibility grouping of Fusarium oxysporum f. sp. vanillae in Indonesia. Indon. J. Crop Science 1994, 9, 29-39.

6. Thomas, J.; Vijayan, A.; Bhai, R. Vanilla disease in India and their management. Indian Journal of Arecanut Spices and Medical Plants. 2002, 4, 143-149.

7. O'Donnell, K.; Ward, T. J.; Robert, V. A. R.; Crous, P. W.; Geiser, D. W.; Kang S. DNA sequence-based identification of Fusarium: current status and future directions. Phytoparasitica 2015, 43, 583-595. Doi: https://doi.org/10.1007/s12600-015-0484-z

8. Edel-Hermann, V.; Lecomte, C. Current Status of Fusarium oxysporum Formae Speciales and Races. Phytopathol. 2019, 109, 512-530. Doi: https://doi.org/10.1094/PHYTO-08-18-0320-RVW 
9. Ma, L.; Van der Does, C.; Borkovich, K.; Coleman, J.; Daboussi, M.; Di Pietro, A.; Dufresne, M.; Freitag, M.; Grabherr, M.; Henrissat, B.; Houterman, P.; Kang, S.; Shim, W.; Woloshuk, C.; Xie, X.; Xu, J.; Antoniw, J.; Baker, S.; Bluhm, B.; Breakspear, A.; Brown, D.; Butchko, R.; Chapman, S.; Coulson, R.; Coutinho, P.; Danchin, E.; Diener, A.; Gale, L.; Gardiner, D.; Goff, S.; Hammond-Kosack, K.; Hilburn, K.; Hua-Van, A.; Jonkers, W.; Kazan, K.; Kodira, C.; Koehrsen, M.; Kumar, L.; Lee, Y.; Li, L.; Manners, J.; Miranda-Saavedra, D.; Mukherjee, M.; Park, G.; Park, J.; Park, S.; Proctor, R.; Regev, A.; Ruiz-Roldan, M.; Sain, D.; Sakthikumar, S.; Sykes, S.; Schwartz, D.; Turgeon, B.; Wapinski, I.; Yoder, O.; Young, S.; Zeng, Q.; Zhou, S.; Galagan, J.; Cuomo, C.; Kistler, H.; Rep, M. Comparative genomics reveals mobile pathogenicity chromosomes in Fusarium. Nature 2010, 464, 367-373. Doi: https://doi.org/10.1038/nature08850

10. O'Donnell, K.; Kistler, H.; Cigelnik, E.; Ploetz, R. Multiple evolutionary origins of the fungus causing Panama disease of banana: Concordant evidence from nuclear and mitochondrial gene genealogies. PNAS 1998, 95, 2044-2049. Doi: https://doi.org/10.1073/pnas.95.5.2044

11. Taylor, J.; Geiser, D.; Burt, A.; Koufopanou, V. The Evolutionary Biology and Population Genetics Underlying Fungal Strain Typing. Clinical Microbiology Reviews 1999, 12, 126-146. Doi: https://doi.org/10.1128/CMR.12.1.126

12. Pinaria, A.; Liew, E.; Burgess, L. Fusarium species associated with vanilla stem rot in Indonesia. Australasian Plant Pathology 2010, 39, 176-183. https://doi.org/10.1071/AP09079

13. Flores-de la Rosa, F.R.; De Luna, E.; Adame-García, J.; Iglesias-Andreu, L.G.; Luna-Rodríguez, M. Phylogenetic position and nucleotide diversity of Fusarium oxysporum f. sp. vanillae worldwide based on translation elongation $1 \alpha$ factor sequences. Plant Pathology 2018, 67, 1278-1285. Doi: https://doi.org/10.1111/ppa.12847

14. Solano-De la Cruz, M.; Adame-Garcia, J.; Gregorio-Jorge, J.; Jimenez-Jacinto, V.; Vega-Alvarado, L.; Iglesias-Andreu, L.; Escobar-Hernandez, E.; Luna-Rodriguez, M. Functional categorization of de novo transcriptome assembly of Vanilla planifolia Jacks. potentially points to a translational regulation during early stages of infection by Fusarium oxysporum $\mathrm{f}$. sp. vanillae. BMC Genomics 2019, 20, 1-15. Doi: https://doi.org/10.1186/s12864-019-6229-5

15. Andrews, S. FastQC: a quality control tool for high throughput sequence data. 2010. Available online at: http://www.bioinformatics.babraham.ac.uk/projects/fastqc

16. Ewels, P.; Magnusson, M.; Lundin, S.; Kaller, M. MultiQC: summarize analysis results for multiple tools and samples in a single report. Bioinformatics. 2016, 32, 3047-3048. Doi: https://doi.org/10.1093/bioinformatics/btw354

17. Dobin, A.; Davis, C.; Schlesinger, F.; Drenkow, J.; Zaleski, C.; Jha, S.; Batut, P.; Chaisson, M.; Gingeras, T. STAR: ultrafast universal RNA-seq aligner. Bioinformatics. 2013, 29, 15-21. Doi: https://doi.org/10.1093/bioinformatics/bts635

18. Li, H.; Handsaker, B.; Wysoker, A.; Fennell, T.; Ruan, J.; Homer, N.; Marth, G.; Abecasis, G.; Durbin, R. The Sequence Alignment/Map format and SAMtools. Bioinformatics 2009, 25, 2078-2079. Doi: https://doi.org/10.1093/bioinformatics/btp352

19. Kumar, S.; Stecher, G.; Li, M.; Knyaz, C.; Tamura, K. MEGA X: Molecular Evolutionary Genetics Analysis across computing platforms. Molecular Biology and Evolution 2018, 35, 1547-1549. Doi: https://doi.org/10.1093/molbev/msz312

20. Quinlan, A.; Hall, I. BEDTools: a flexible suite of utilities for comparing genomic features. Bioinformatics 2010, 26, 841-842. Doi: https://doi.org/10.1093/bioinformatics/btq033

21. Howe, K.; Contreras-Moreira, B.; De Silva, N.; Maslen, G.; Akanni, W.; Allen, J.; Alvarez-Jarreta, J.; Barba, J.; Bolser, D.; Cambell, L.; Carbajo, M.; Chakiachvili, M.; Christensen, M.; Cummins, C.; Cuzick, A.; Davis, P.; Fexova, S.; Gall, A.; George, N.; Gil, L.; Gupta, P.; Hammond-Kosack, K.; Haskell, E.; Hunt, S.; Jaiswal, P.; Janacek, S.; Kersey, P.; Langridge, N.; Maheswari, U.; Maurel, T.; McDowall, M.; Moore, B.; Muffato, M.; Naamati, G.; Naithani, S.; Olson, A.; Papatheodorou, I.; Patricio, M.; Paulini, M.; Pedro, H.; Perry, E.; Preece, J.; Rosello, M.; Russell, M.; Sitnik, V.; Staines, D.; Stein, J.; Tello-Ruiz, M.; Trevanion, S.; Urban, M.; Wei, S.; Ware, D.; Williams, G.; Yates, A.; Flicek, P. Ensembl Genomes 2020-enabling non-vertebrate genomic research. Nucleic Acids Research 2020, 48: D689-D695. Doi: https://doi.org/10.1093/nar/gkz890

22. Kanehisa, M.; Goto, S. KEGG: Kyoto encyclopedia of genes and genomes. Nucleic Acids Research 2000, 28, 27-30. Doi: https://doi.org/10.1093/nar/28.1.27

23. Alexa, A.; Rahnenführer, J.; Lengauer, T. Improved scoring of functional groups from gene expression data by decorrelating GO graph structure. Bioinformatics 2006, 22, 1600-1607. Doi: https://doi.org/10.1093/bioinformatics/btl140

24. Wickham, H. ggplot2: Elegant Graphics for Data Analysis. Springer-Verlag New York. ISBN 978-3-319-24277-4, 2016. Doi: https://ggplot2.tidyverse.org

25. Yu, G.; Wang, L.; Han, Y.; He, Q. clusterProfiler: An R Package for Comparing Biological Themes Among Gene Clusters. OMICS 2012, 16, 284-287. Doi: https://doi.org/10.1089/omi.2011.0118

26. Jones, P.; Binns, D.; Chang, H.; Fraser, M.; Li, W.; McAnulla, C.; McWilliam, H.; Maslen, J.; Mitchell, A.; Nuka, G.; Pesseat, S.; Quinn, A.; Sangrador-Vegas, A.; Scheremetjew, M.; Yong, S.; Lopez, R.; Hunter, S. InterProScan 5: genome-scale protein function classification. Bioinformatics 2014, 30, 1236-1240. Doi: https://doi.org/10.1093/bioinformatics/btu031

27. Leslie, J.F., Pearson, C.A.S., Nelson, P.E., Toussoun, T.A. Fusarium spp. from corn, sorghum, and soybean fields in the central and eastern United States. Phytopathol. 1990, 80, 343-350.

28. Bacon, C.W.; Yates I.E. Endophytic Root Colonization by Fusarium Species: Histology, Plant Interactions, and Toxicity. In: Schulz B.J.E., Boyle C.J.C., Sieber T.N. (eds) Microbial Root Endophytes. Soil Biology, vol 9. Springer, Berlin, Heidelberg. 2006. Doi: https://doi.org/10.1007/3-540-33526-9_8

29. Flores-de la Rosa, F.R.; Jiménez-Gasco, M.del M.; Adame-García, J.; Rivera-Fernández, A.; Luna-Rodriguez, M. Genetic Comparison of Pathogenic and Non-Pathogenic Fusarium Oxysporum Endophytes from Vanilla sp. in Mexico. Research Square 2021. Doi: : https://doi.org/10.21203/rs.3.rs-217974/v1 
30. Stone, J.K.; Bacon, C.W.; White, J.F. An Overview of Endophytic Microbes: Endophytism Defined. In: Bacon, C.W. and White, J.F., Eds., Microbial Endophytes, Marcel Dekker, New York, 2000, 3-29.

31. Khoyratty, S.; Dupont, J.; Lacoste, S.; Palama, T.L.; Choi, Y.H.; Kim, H.K.; Payet, B.; Grisoni, M; Fouillaud, M.; Verpoorte, R.; Kodja, H. Fungal endophytes of Vanilla planifolia across Réunion Island: isolation, distribution and biotransformation. BMC Plant Biol. 2015, 15, 142. Doi: https://doi.org/10.1186/s12870-015-0522-5

32. de Lamo, F. J.; Takken, F. L. W. Biocontrol by Fusarium oxysporum Using Endophyte-Mediated Resistance. Front. Plant Sci. 2020, 11,37. Doi: https://doi.org/10.3389/fpls.2020.00037

33. Jonkers, W.; Rodrigues, C.D.; Rep M. Impaired colonization, and infection of tomato roots by the Deltafrp1 mutant of Fusarium oxysporum correlates with reduced CWDE gene expression. Mol Plant Microbe Interact. 2009, 22, 507-18. Doi: https://doi.org/10.1094/MPMI-22-5-0507

34. van Dam, P.; Fokkens, L.; Ayukawa, Y.; van der Gragt, M.; ter Horst, A.; Brankovics, B.; Houterman, P.M.; Arie, T.; Rep, M. A mobile pathogenicity chromosome in Fusarium oxysporum for infection of multiple cucurbit species. Sci. Rep. 2017, 7. Doi: https://doi.org/10.1038/s41598-017-07995-y

35. Schmidt, S. M.; Houterman, P. M.; Schreiver, I.; Ma, L. S.; Amyotte, S.; Chellappan, B.; Boeren, S.; Takken, F.L.W.; Rep, M. MITEs in the promoters of effector genes allow prediction of novel virulence genes in Fusarium oxysporum. BMC Genomics 2013, 14. Doi: https://doi.org/10.1186/1471-2164-14-119

36. Rep, M.; van der Does; H. C.; Meijer, M.; van Wijk, R.; Houterman, P. M.; Dekker, H. L.; de Koster, C.G.; Cornelissen, B.J.C. A small, cysteine-rich protein secreted by Fusarium oxysporum during colonization of xylem vessels is required for I-3-mediated resistance in tomato. Mol. Microbiol. 2004, 53, 1373-1383. Doi: https://doi.org/10.1038/10.1111/j.1365-2958.2004.04177.x

37. Di, X. T.; Cao, L. X.; Hughes, R. K.; Tintor, N.; Banfield, M. J.; Takken, F. L. W. Structure function analysis of the Fusarium oxysporum Avr2 effector allows uncoupling of its immune suppressing activity from recognition. New Phytol. 2017, 216 (3), 897-914. Doi: https://doi.org/10.1111/nph.14733

38. Houterman, P. M.; Cornelissen, B. J.; Rep, M. Suppression of plant resistance gene-based immunity by a fungal effector. PloS Pathog. 2008, 4 (5), e1000061. Doi: https://doi.org/10.1371/journal.ppat.1000061

39. Gawehns, F.; Houterman, P. M.; Ichou, F. A.; Michielse, C. B.; Hijdra, M.; Cornelissen, B. J. C.; Rep, M.; Takken, F.L.W. The Fusarium oxysporum effector six6 contributes to virulence and suppresses I-2 mediated cell death. Mol. Plant-Microb. Interact. 2014, 27 (4), 336-348. doi: https://doi.org/10.1094/MPMI-11-13-0330-R

40. Sharma, M.; Sengupta, A.; Ghosh, R.; Agarwal, G.; Tarafdar, A.; Nagavardhini, A.; Pande, S.; Varshney, R. K. Genome wide transcriptome profiling of Fusarium oxysporum f. sp. ciceris conidial germination reveals new insights into infection-related genes. 2016, Sci Rep 6, 37353. Doi: https://doi.org/10.1038/srep37353

41. Phillips, D.A.; Fox, T.C.; King, M.D.; Bhuvaneswari, T.V.; Teuber, L.R. Microbial Products Trigger Amino Acid Exudation from Plant Roots. Plant Physiology 2004, 136, 2887-2894. Doi: https://doi.org/10.1104/pp.104.044222

42. Jonkers, W.; Rodrigues, C.D.; Rep M. Impaired colonization, and infection of tomato roots by the Deltafrp1 mutant of Fusarium oxysporum correlates with reduced CWDE gene expression. Mol Plant Microbe Interact. 2009, 22, 507-18. Doi: https://doi.org/10.1094/MPMI-22-5-0507

43. Scandiani, M.M.; Luque, A.G.; Razori, M.V.; Casalini, C.L.; Aoki, T.; O'Donnell, K.; Cervigni, G.D.L.; Spampinato, C.P. Metabolic profiles of soybean roots during early stages of Fusarium tucumaniae infection. Journal of Experimental Botany 2015, 66402,. Doi: https://doi.org/10.1093/jxb/eru432

44. Tchameni, N.S.; Nwaga, D.; Nana, W.L.; Ngonkeu, M.E.L.; Fokom, R.; Etoa, F.X. Growth enhancement, amino acid synthesis and reduction in susceptibility towards Phytophthora megakarya by Arbuscular Mycorrhizal Fungi inoculation in cocoa plants. J. Phytopathol. 2012, 160, 220-228. Doi: https://doi.org/10.1111/j.1439-0434.2012.01888.x

45. Okada, T.; Matsubara, Y. Tolerance to Fusarium Root Rot and the Changes in Free Amino Acid Contents in Mycorrhizal Asparagus Plants, HortScience 2012, 47, 751-754. Doi: https://journals.ashs.org/hortsci/view/journals/hortsci/47/6/article-p751.xml

46. Jastrzębowska, K.; Gabriel, I. Inhibitors of amino acids biosynthesis as antifungal agents. Amino Acids 2015, 47, 227-249. Doi: https://doi.org/10.1007/s00726-014-1873-1

47. Boedi, S.; Berger, H.; Sieber, C.; Münsterkötter, M.; Maloku, I.; Warth, B.; Sulyok, M.; Lemmens, M.; Schuhmacher, R.; Güldener, U.; Strauss, J. Comparison of Fusarium graminearum Transcriptomes on Living or Dead Wheat Differentiates Substrate-Responsive and Defense-Responsive Genes. Frontiers in Microbiology 2016, 7, 1-24. Doi: https://doi.org/10.3389/fmicb.2016.01113

48. Liu, T.B.; Xue, C. The Ubiquitin-Proteasome System and F-box Proteins in Pathogenic Fungi. Mycobiology 2011, 39, 243-8. Doi: https://doi.org/10.5941/MYCO.2011.39.4.243

49. Lv, W.; Wang, C.; Yang, N.; Que, Y.; Talbot, N.J.; Wang, Z. Genome-wide functional analysis reveals that autophagy is necessary for growth, sporulation, deoxynivalenol production and virulence in Fusarium graminearum. Sci Rep 2017, 7, 11062. Doi: https://doi.org/10.1038/s41598-017-11640-z

50. Khalid, A.R.; Lv, X.; Naeem, M.; Mehmood, K.; Shaheen, H.; Dong, P.; Qiu, D.; Ren, M. Autophagy Related Gene (ATG3) is a Key Regulator for Cell Growth, Development, and Virulence of Fusarium oxysporum. Genes 2019, $10,658$. https://doi.org/10.3390/genes10090658

51. Pan, J.; Hu, C.; Yu, J.-H. Lipid Biosynthesis as an Antifungal Target. J. Fungi 2018, 4, 50. https://doi.org/10.3390/jof4020050

52. Bani, M.; Pérez-De-Luque, A.; Rubiales, D.; Rispail, N. Physical, and chemical barriers in root tissues contribute to quantitative resistance to Fusarium oxysporum f. sp. pisi in pea. Front Plant Sci. 2018, 9, 1-16. Doi: https://doi.org/10.3389/fpls.2018.00199 
53. Olivain, C.; Alabouvette, C. Process of tomato root colonization by a pathogenic strain of Fusarium oxysporum f. sp. lycopersici in comparison with a non-pathogenic strain. New Phytologist 1999, 141: $497-510 . \quad$ Doi: https://doi.org/10.1046/j.1469-8137.1999.00365.x

54. Schneider, E.; Carlquist, S. Pit membrane remnants in perforation plates and other vessel details of cornales. Brittonia 2004, 56, 275-283. https://doi.org/10.1663/0007

55. Groenewald S. Biology, pathogenicity, and diversity of Fusarium oxysporum f. sp. cubense. Master of Science dissertation. Pretoria University 2006.

56. Olivain, C.; Humbert, C.; Nahalkova, J.; Fatehi, J.; L'Haridon, F.; Alabouvette, C. Colonization of tomato root by pathogenic and nonpathogenic Fusarium oxysporum strains inoculated together and separately into the soil. Applied and Environmental Microbiology 2006, 72, 1523-1531. Doi: https://doi.org/10.1128/AEM.72

57. Koyyappurath, S.; Conéjéro, G.; Dijoux, J., Lapeyre-Montés, F.; Jade, K.; Chiroleu, F.; Gitaneae, F.; Verdeil, J.; Besse, P.; Grisoni, M. Differential responses of Vanilla accessions to root rot and colonization by Fusarium oxysporum f. sp. radicis-vanillae. Frontiers in Plant Science 2015, 6: 1-16. Doi: https://doi.org/10.3389/fpls.2015.01125

58. Koyyappurath, S.; Atuahiva, T.; Guen, L.; Batina, H.; Le Squin, S.; Gautheron, N.; Hermann, V.; Peribe, J.; Jahiel, M.; Steinberg, C.; Liew, E.; Alabouvette, C.; Besse, P.; Dron, M.; Sache, I.; Laval, V.; Grisoni M. Fusarium oxysporum f. sp. radicis-vanillae is the causal agent of root and stem rot in vanilla. Plant Pathology 2016, 65, 612-625. Doi: https://doi.org/10.1111/ppa.12445

59. Ma, L.; Geiser, D.; Proctor, R.; Rooney, A.; O’Donnell, K.; Trail, F.; Gardiner, D.; Manners, J.; Kazan, K. Fusarium Pathogenomics. Annual Reviews in Microbiology 2013, 67, 399-416. Doi: https://doi.org/10.1146/annurev-micro-092412-155650 